\title{
High Intra- and Inter-Tumoral Heterogeneity of $R A S$ Mutations in Colorectal Cancer
}

\author{
Marion Jeantet 1,2,3, David Tougeron 1,4,5, Gaelle Tachon ${ }^{1,2}$, Ulrich Cortes 1,2, \\ Céline Archambaut 1,2 , Gaelle Fromont ${ }^{3}$ and Lucie Karayan-Tapon 1,2,6,* \\ 1 Faculté de Médecine Pharmacie, Université de Poitiers, 86021 Poitiers, France; marion_doc@hotmail.fr (M.J.); \\ david.tougeron@chu-poitiers.fr (D.T.); gaelle.tachon@chu-poitiers.fr (G.T.); \\ ulrich.cortes@chu-poitiers.fr (U.C.); labonco@gmail.com (C.A.) \\ 2 Département de Cancérologie Biologique, Centre Hospitalo-Universitaire de Poitiers, 86021 Poitiers, France \\ 3 Département d'anatomopathologie, Centre Hospitalo-Universitaire de Poitiers, 86021 Poitiers, France; \\ Gaelle.fromont-hancard@univ-tours.fr \\ 4 Département de Gastroentérologie, Centre Hospitalo-Universitaire de Poitiers, 86021 Poitiers, France \\ 5 Laboratoire Inflammation, Tissus Epithéliaux et Cytokines, EA 4331, Université de Poitiers, \\ 86021 Poitiers, France \\ 6 INSERM1084, Laboratoire de Neurosciences Expérimentales et Cliniques, Université de Poitiers, \\ 86021 Poitiers, France \\ * Correspondence: 1.karayan-tapon@chu-poitiers.fr; Tel.: +33-5-4944-4988 \\ Academic Editor: William Chi-shing Cho \\ Received: 5 September 2016; Accepted: 25 November 2016; Published: 1 December 2016
}

\begin{abstract}
Approximately $30 \%$ of patients with wild type RAS metastatic colorectal cancer are non-responders to anti-epidermal growth factor receptor monoclonal antibodies (anti-EGFR mAbs), possibly due to undetected tumoral subclones harboring RAS mutations. The aim of this study was to analyze the distribution of RAS mutations in different areas of the primary tumor, metastatic lymph nodes and distant metastasis. A retrospective cohort of 18 patients with a colorectal cancer (CRC) was included in the study. Multiregion analysis was performed in 60 spatially separated tumor areas according to the pathological tumor node metastasis (pTNM) staging and KRAS, NRAS and BRAF mutations were tested using pyrosequencing. In primary tumors, intra-tumoral heterogeneity for $R A S$ mutation was found in 33\% of cases. Inter-tumoral heterogeneity for RAS mutation between primary tumors and metastatic lymph nodes or distant metastasis was found in $36 \%$ of cases. Moreover, $28 \%$ of tumors had multiple RAS mutated subclones in the same tumor. A high proportion of CRCs presented intra- and/or inter-tumoral heterogeneity, which has relevant clinical implications for anti-EGFR mAbs prescription. These results suggest the need for multiple RAS testing in different parts of the same tumor and/or more sensitive techniques.
\end{abstract}

Keywords: colorectal cancer; $R A S$ mutation; intra-tumoral heterogeneity; inter-tumoral heterogeneity

\section{Introduction}

Colorectal cancer (CRC) is the third deadliest of all cancers [1]. Nearly one-third of the patients will eventually die of the disease. Targeting the epidermal growth factor receptor (EGFR), an important component in CRC carcinogenesis, is one of the major therapeutic options in metastatic CRC (mCRC). Two anti-EGFR monoclonal antibodies (mAbs), cetuximab and panitumumab, are commonly used in mCRC. Clinical trials have shown the benefit of anti-EGFR mAbs alone or in combination with chemotherapy in mCRC [2-4].

Several studies have demonstrated that KRAS mutation in exon 2 is a predictive marker of resistance to anti-EGFR mAbs [5]. More recently, other activating RAS mutations (KRAS exons 3 and 4 and NRAS exons 2, 3 and 4) were also shown to confer resistance to anti-EGFR mAbs $[3,4]$. 
Approximately $50 \%$ of mCRC harbor mutations in exons 2, 3 or 4 of either KRAS or NRAS genes [6]. The most frequent mutations are detected in exon 2 (codons 12 and 13) of KRAS (40\%), and, to a lesser extent, in exon 3 (codons 59 and 61 ) and exon 4 (codons 117 and 146) of $K R A S(\approx 7 \%$ of cases). Activating mutations of NRAS occur only in a subset of $\mathrm{mCRC}(\approx 5 \%$ of cases), mostly at codons 12 , 13 and 61 [6]. The BRAF $F^{V 600 E}$ mutation occurs in $10 \%-15 \%$ of mCRC [7,8]. BRAF $F^{V 600 E}$ mutant mCRC is associated with poorer outcomes. However, whether this mutation is predictive of resistance to anti-EGFR $m A$ bs is uncertain [7].

Only wild-type (WT) RAS mCRCs benefit from treatment with anti-EGFR mAbs. Nevertheless, nearly $35 \%$ of patients with WT RAS tumors do not respond to anti-EGFR treatment $[3,4,6]$. Several molecular mechanisms underlying the development of treatment resistance have been reported in the literature [9]. One possible explanation lies in tumor heterogeneity with regard to $R A S$ mutations $[8,10]$. There is a general consensus that progression of cancer develops from a single mutated cell, followed by clonal expansion associated with genetic alterations. The acquisition of these alterations can result in the emergence of new tumor subclones with different genotypes [11]. Intra-tumoral heterogeneity is defined by the presence of at least two different tumoral subclones within the same tumor mass. Inter-tumoral heterogeneity consists in the presence of at least two different tumor subclones at different tumor sites in a single patient (i.e., primary tumor, metastatic lymph nodes or metastases) [12]. Both intra- and inter-tumoral heterogeneity are important to identify since they could affect response to targeted therapies.

Different levels of tumoral heterogeneity have already been observed in several tumor types [13-15]. Nevertheless, there are few data concerning intra- and inter-tumoral heterogeneity in CRC. KRAS, NRAS and BRAF mutations are considered to be mutually exclusive in CRC [16]. Inter-tumoral heterogeneity seems to be relatively low between primary and metastatic lesions in mCRC since concordance of KRAS and BRAF status is over 95\% [17-19]. Nevertheless, these previous works used sequencing methods with low sensitivity and did not study complete RAS status. In addition, few data have been available concerning inter-tumoral heterogeneity of $R A S$ and $B R A F$ mutations between primary tumors and lymph node metastasis. Data concerning intra-tumoral heterogeneity of $R A S$ and $B R A F$ mutations between different areas of primary tumor data are lacking. In the present study, we investigated intra- and inter-tumoral heterogeneity of $R A S$ and BRAF mutations in 60 tumor areas from 18 CRCs.

\section{Results}

\subsection{Population}

We retrospectively analyzed tumors from 18 patients with CRC (twelve colons and six rectums). Mean age at diagnosis was $66.5 \pm 9.0$ years (Table 1$)$. Tumor stages were stage I $(n=1,5 \%)$, stage II $(n=3,17 \%)$, stage III $(n=5,28 \%)$ and stage IV $(n=9,50 \%)$. According to the pathological tumor node metastasis (pTNM) staging, tumors were pT2 $(n=1,5 \%)$, pT3 $(n=14,78 \%)$ and pT4a $(n=3,17 \%)$.

Tumors were assessed for KRAS mutations in codons 12 and $13\left(K R A S^{12-13}\right)$ on the initial transparietal section (ITS). Five tumors were $K R A S^{12-13}$ mutant (MUT), seven tumors were $K R A S^{12-13}$ WT and six tumors had potential low level (PLLM) KRAS $S^{12-13}$ mutations (Table 2). Tumors with PLLM are defined as tumors with mutant allele frequency $\geq$ limit of detection (LOD) and $\leq$ LOD $+3 \%$ as described in the materials and methods section [8]. One WT KRAS tumor had a BRAFV600E mutation. 
Table 1. Patients and tumor characteristics.

\begin{tabular}{|c|c|c|c|c|c|c|c|c|c|c|}
\hline Patient & Age & Sex & Tumor Site & Stage & pTNM 2009 & Initial KRAS & Initial $B R A F$ & Recurrence & OS & Status \\
\hline 2 & 77.9 & $\mathrm{~F}$ & right colon & III & pT3N1bM0 & WT & WT & yes & 48.68 & dead \\
\hline 3 & 75.9 & M & left colon & III & pT4aN2bM0 & WT & WT & no & 2.50 & alive \\
\hline 4 & 79.2 & $\mathrm{~F}$ & right colon & II & pT3N0M0 & WT & V600E & no & 59.14 & alive \\
\hline 6 & 55.9 & M & left colon & I & pT2N0M0 & WT & WT & no & 62.50 & alive \\
\hline 7 & 67.3 & M & left colon & III & pT3N1bM0 & G12S & WT & yes & 69.21 & dead \\
\hline 8 & 72.9 & $\mathrm{~F}$ & left colon & IV & pT3N1bM1 & G12V & WT & - & 26.35 & dead \\
\hline 9 & 58.5 & M & left colon & III & pT4aNOM0 & G12S & WT & yes & 82.86 & dead \\
\hline 12 & 77.8 & $\mathrm{~F}$ & left colon & IV & pT4aN0M1 & G12D & WT & - & 22.60 & dead \\
\hline 14 & 56.4 & $\mathrm{~F}$ & right colon & IV & pT3N1aM1 & G12V & WT & - & 48.39 & dead \\
\hline 15 & 77.7 & M & left colon & IV & pT3N2bM1 & G12D & WT & - & 14.41 & dead \\
\hline 16 & 74.7 & $\mathrm{~F}$ & right colon & II & pT3N0M0 & G12V & WT & no & 37.70 & alive \\
\hline 13 & 66.0 & $\mathrm{M}$ & left colon & II & pT3N0M0 & G12V & WT & - & 49.00 & alive \\
\hline 1 & 61.9 & M & rectum & IV & pT3N2bM1 & WT & WT & - & 22.5 & dead \\
\hline 5 & 58.9 & M & rectum & IV & pT3N0M1 & WT & WT & - & 52.34 & dead \\
\hline 10 & 57.1 & $\mathrm{~F}$ & rectum & IV & pT3N2bM1 & WT & WT & - & 44.80 & dead \\
\hline 11 & 65.2 & $\mathrm{~F}$ & rectum & IV & pT3N2bM1 & G12D & WT & - & 17.96 & dead \\
\hline 17 & 54.9 & $\mathrm{~F}$ & rectum & III & pT3N2aM0 & G12V & WT & yes & 41.22 & alive \\
\hline 18 & 58.6 & M & rectum & IV & pT3N0M1 & G12R & WT & - & 43.82 & dead \\
\hline
\end{tabular}

M: male; F: female; OS: overall survival; WT: wild-type; pTNM: pathological tumor node metastasis. 
Table 2. RAS and BRAF mutations in the initial transparietal sections $(n=13)$ and spatially separated tumor areas $(n=43)$ in 13 colorectal cancers.

\begin{tabular}{|c|c|c|c|c|c|}
\hline Heterogeneity & Patients & pTNM & $\%$ TC & KRAS & Other Genes \\
\hline \multirow{11}{*}{$\begin{array}{l}\text { Intra-tumoral } \\
\text { heterogeneity }\end{array}$} & \multirow{3}{*}{4} & ITS & 70 & WT & $B R A F: V 600 E$ (PLLM) \\
\hline & & pT1 & 30 & WT & WT \\
\hline & & pT2 & 90 & WT & $B R A F: \mathrm{V} 600 \mathrm{E}$ (MUT) \\
\hline & \multirow{4}{*}{9} & ITS & 70 & G12S (PLLM)/Q61R (PLLM) & NRAS:Q61R (MUT) \\
\hline & & pT1 & 95 & WT & NRAS:Q61R (MUT) \\
\hline & & pT2 & 70 & WT & NRAS:Q61R (MUT) \\
\hline & & pT3 & 25 & WT & NRAS:Q61R (MUT) \\
\hline & \multirow{4}{*}{18} & ITS & 80 & G12R (MUT) & WT \\
\hline & & pT1 & 30 & G12R (MUT) & NRAS : K117N (PLLM) \\
\hline & & pT2 & 35 & G12R (MUT) & WT \\
\hline & & pT3 & 20 & G12R (MUT) & WT \\
\hline \multirow{13}{*}{$\begin{array}{l}\text { Intra-tumoral and } \\
\text { Inter-tumoral } \\
\text { heterogeneity }\end{array}$} & \multirow{4}{*}{2} & ITS & 80 & WT & NRAS: A59T (PLLM) \\
\hline & & pT2 & 15 & WT & WT \\
\hline & & pT3 & 20 & G13D (MUT) & WT \\
\hline & & $\mathrm{N}$ & 5 & WT & WT \\
\hline & \multirow{5}{*}{7} & ITS & 60 & G12S (PLLM) & FA (NRAS61 et KRAS146) \\
\hline & & pT1 & 70 & G12D (MUT)/Q61L (PLLM) & NRAS:Q61K (MUT) \\
\hline & & pT2 & 25 & G12S (MUT) & NRAS:Q61K (MUT) \\
\hline & & pT3 & 40 & Q61L (PLLM) & NRAS:Q61K (MUT) \\
\hline & & $\mathrm{N}$ & 25 & WT & NRAS:Q61K (MUT) \\
\hline & \multirow{4}{*}{12} & ITS & 40 & G12D (PLLM)/A146T (MUT) & WT \\
\hline & & pT2 & 15 & $\mathrm{~A} 146 \mathrm{~T}(\mathrm{MUT})$ & WT \\
\hline & & pT3 & 20 & Q61H(PLLM)/A146T (MUT) & FA $(B R A F)$ \\
\hline & & $\mathrm{M}$ & 25 & WT & FA(NRAS59) \\
\hline \multirow{5}{*}{$\begin{array}{c}\text { Inter-tumoral } \\
\text { heterogeneity only }\end{array}$} & \multirow{5}{*}{14} & ITS & 30 & G12V (MUT) & WT \\
\hline & & pT1 & 5 & G12V (PLLM) & WT \\
\hline & & pT2 & 40 & G12V (MUT) & WT \\
\hline & & pT3 & 5 & G12V (PLLM) & WT \\
\hline & & $\mathrm{N}$ & 5 & WT & WT \\
\hline \multirow{27}{*}{$\begin{array}{l}\text { Mutation without } \\
\text { heterogeneity }\end{array}$} & \multirow{5}{*}{8} & ITS & 30 & G12V (MUT) & WT \\
\hline & & pT1 & 60 & G12V (MUT) & WT \\
\hline & & pT2 & 75 & G12V (MUT) & WT \\
\hline & & pT3 & 60 & G12V (MUT) & WT \\
\hline & & $\mathrm{N}$ & 80 & G12V (MUT) & WT \\
\hline & \multirow{4}{*}{11} & ITS & 5 & G12D (PLLM) & WT \\
\hline & & pT2 & 5 & G12D (MUT) & WT \\
\hline & & pT3 & 5 & G12D (PLLM) & WT \\
\hline & & $\mathrm{N}$ & 5 & G12D (MUT) & WT \\
\hline & \multirow{4}{*}{13} & ITS & 70 & G12V (MUT) & WT \\
\hline & & pT1 & 70 & G12V (MUT) & WT \\
\hline & & pT2 & 10 & G12V (MUT) & WT \\
\hline & & pT3 & 15 & G12V (MUT) & WT \\
\hline & \multirow{5}{*}{15} & ITS & 40 & G12D (MUT) & WT \\
\hline & & pT1 & 60 & G12D (MUT) & WT \\
\hline & & pT2 & 15 & G12D (MUT) & WT \\
\hline & & pT3 & 20 & G12D (MUT) & WT \\
\hline & & $\mathrm{N}$ & 40 & G12D (MUT) & WT \\
\hline & \multirow{4}{*}{16} & ITS & 40 & G12V (MUT) & WT \\
\hline & & pT1 & 80 & G12V (MUT) & WT \\
\hline & & pT2 & 40 & G12V (MUT) & WT \\
\hline & & pT3 & 10 & G12V (MUT) & WT \\
\hline & \multirow{5}{*}{17} & ITS & 30 & G12V (MUT) & WT \\
\hline & & pT1 & 30 & G12V (MUT) & WT \\
\hline & & pT2 & 20 & G12V (MUT) & WT \\
\hline & & pT3 & 10 & G12V (MUT) & WT \\
\hline & & $\mathrm{N}$ & 20 & G12V (MUT) & WT \\
\hline
\end{tabular}

\%TC: percentage of tumoral cells; ITS: initial transparietal section; N: metastatic lymph node; M: metastasis;

WT: wild-type; PLLM: potential low level mutation; MUT: mutant; FA: failed analysis.

\subsection{Intra-Tumoral and Inter-Tumoral Heterogeneity of KRAS and NRAS Mutations}

Thirty-nine percent of mCRC studied harbored intra- and/or inter-tumoral heterogeneity (Table 2).

Among the six tumors with intra-tumoral heterogeneity, only two were KRAS ${ }^{12-13}$ WT in ITS (cases 2 and 4). Three tumors (cases 2, 7, 12) presented KRAS ${ }^{12-13}$ PLLM or MUT in at least one of the tumor areas selected, but they also presented other KRAS or NRAS MUT or PLLM in other areas of 
the tumor (case 2) or in the same area (case 7, 12). An additional NRAS ${ }^{117}$ PLLM in the submucosa (pT1) was observed for one tumor (case 18) (Table 2 and Figure S1). All in all, 33\% of tumors (6/18) showed intra-tumoral heterogeneity for RAS mutation. The others presented either a WT pattern (cases 1, 3, 5, 6 and 10) or the same mutation with variation in allele frequency (cases 8, 11,13-17) for all intra-tumoral areas (Table 2 and Table S1).

To evaluate the inter-tumoral heterogeneity, we tested the metastatic lymph nodes and distant metastases for KRAS, NRAS and BRAF mutations when available. Among 11 cases analyzed, four presented inter-tumoral heterogeneity (36\%). Three mutated primary tumors were WT in the metastatic lymph nodes $(\mathrm{N}+)$ or in the visceral metastases $(\mathrm{M}+)$ (cases 2, 12, 14) (Table 2).

It is worth noting that all patients with mCRC with RAS PLLM and/or RAS mutation in a limited area of the tumor (i.e., intra- or inter-tumoral heterogeneity) treated with anti-EGFR mAbs had disease progression at $2-3$ months $(n=5$, cases $2,7,9,11$ and 12$)$.

\subsection{Mutational Intra-Tumoral Heterogeneity}

We focus on mutational intra-tumoral heterogeneity, defined as subclones with different mutant allele frequencies. Therefore, we calculated mutant allele frequency in neoplastic cells (MAFnc) and heterogeneity score (HS) for cases 7-9 and 13-18, which harbored the same mutation from the submucosa (pT1), the muscular layer (pT2) to the subserosa (pT3) (Figure 1). MAFnc is the mutant allele frequency normalized to $100 \%$ tumoral cells and HS corresponds to the fraction of neoplastic cells carrying a specific mutation and was calculated assuming that somatic mutations are usually heterozygous events [20]. Mean HS increased with T stage, $128.4 \pm 46.1$ standard deviation (SD) in pT1, 234.1 \pm 186.8 SD in pT2 and 296.2 \pm 156.9 SD in pT3 $(p=0.03)$ (Figure S2). We observed that HS was higher in pT3 areas in 55\% of cases $(n=5 / 9)$ regardless of the mutation. Mean total HS (sum of HS for each mutation in one tumor) was $692.6 \pm 262.6$ SD. Total HS score was associated neither with tumor stage $(p=0.78)$, nor with tumor location $(p=0.90)$, nor with tumor recurrence $(p=0.56)$.

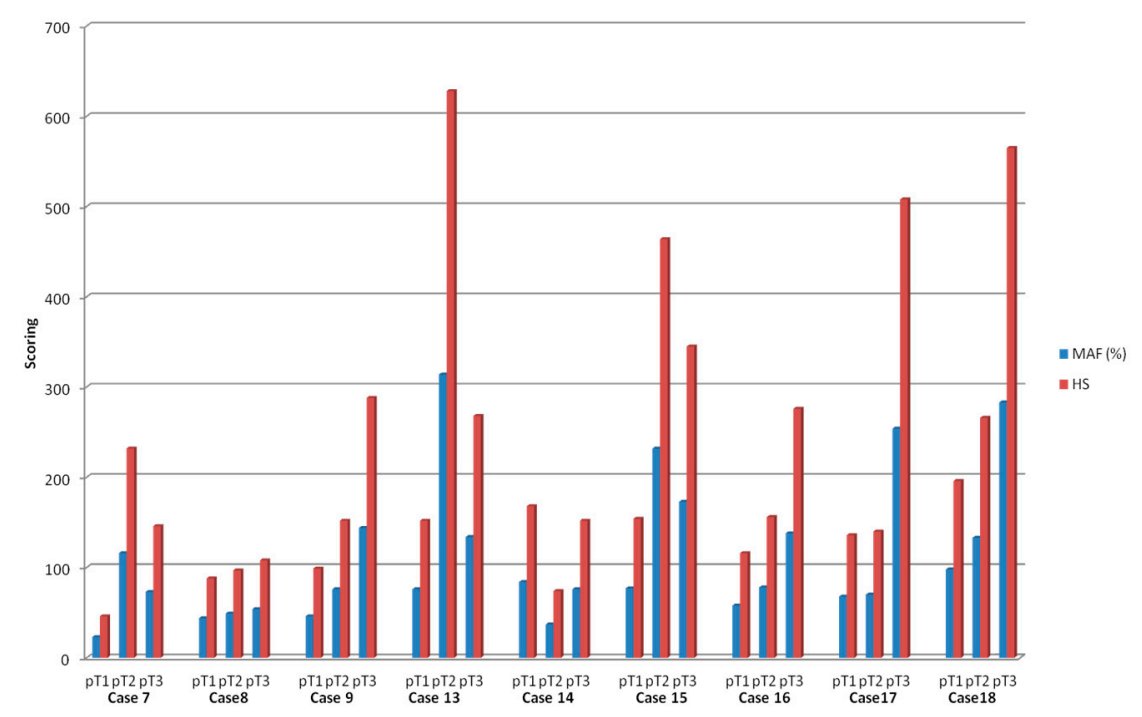

Figure 1. Mutation allele frequency and heterogeneity score. Variability of mutation allele frequency and heterogeneity score between tumoral zone selections in cases which harbored the same mutation in PT1 to pT3. MAF: mutation allele frequency; HS: heterogeneity score.

\subsection{RAS Mutations Are Not Exclusive}

$R A S$ mutations are considered to be mutually exclusive. Only a few articles have reported cases of coexisting KRAS and NRAS mutations [21]. However, in our study, we observed that different KRAS mutations as well as KRAS and NRAS mutations may coexist. Five tumors had multiple RAS mutated subclones in the same tumor (28\%). Moreover, four presented, in one of their tumor selections, at least 
two RAS mutated clones (22\%) (cases 7, 9, 12, and 18) (Table 2). Indeed, we observed the coexistence of different KRAS mutations in three tumors $(17 \%)$ (cases $7,9,12)$ as well as KRAS and NRAS mutations in three tumors (17\%) (cases 7, 9 and 18$)$.

Tumors with two or more RAS mutations (cases 2, 7, 9, 12, and 18) as compared with tumors showing one RAS mutation (cases $8,11,13$ to 17 ) were associated neither with tumor stage $(p=0.29)$ nor with tumor recurrence $(p=0.07)$.

\section{Discussion}

This study revealed a high proportion of intra- and inter-tumoral heterogeneity for RAS mutations in metastatic colorectal cancer. Nearly $40 \%$ of the mCRC studied harbored intra- and/or inter-tumoral heterogeneity. We also demonstrated the coexistence of different $R A S$ mutations within the same tumor. These results have relevant clinical implications for anti-EGFR monoclonal antibodies prescription in mCRC since RAS mutations confer resistance to this treatment. Hence, testing of KRAS and NRAS mutations (codons 12, 13, 59, 61, 117 and 146) is a prerequisite for anti-EGFR mAbs used in mCRC. In daily practice, this testing usually relies on a single tumor sample with high tumor cell content. However, some patients with RAS WT tumor have primary resistance to anti-EGFR mAbs. One explanation is the limited sensitivity of testing methods, leading to false negative results $[6,8,10]$. Nevertheless, intra- and/or inter-tumoral heterogeneity of RAS mutations could be the most important cause of therapeutic failure.

Indeed, recently, the "Big Bang" model emphasized that clonal alterations and subclonal events are an early event in CRC carcinogenesis with subclone mixing [22]. Several neoplastic subclones with co-existing mutations in different genes (as well as different molecular alterations) could be present in a single primary tumor with different mutant allele frequencies [19,20,23-25]. In our study, by testing KRAS and NRAS mutations in histologically relevant macrodissected zones according to PTNM, we observed the presence of (i) mutational intra-tumor heterogeneity with at least two co-existing KRAS and/or NRAS mutations within the same tumor areas and (ii) spatial intra-tumoral heterogeneity with coexistence within the same tumor of KRAS and/or NRAS mutated zones and WT zones. This intra-tumoral heterogeneity was found in $33 \%$ of cases in our study. Kosmidou et al. reported similar results ( $44 \%$ ) of intra-tumoral heterogeneity for KRAS mutations when they compared tumor center and tumor periphery [23]. Recently, Kim et al. observed a substantial level of intra-tumoral heterogeneity ( $46 \%$ to $80 \%$ ) on multiregion biopsies from five mCRCs [26]. Up until now, our study is one of the largest study concerning intra-tumoral heterogeneity and the only one concerning an extended $R A S$ status.

KRAS, NRAS and BRAF mutations are considered to be mutually exclusive in CRC [16]. Nevertheless, in our study, we observed the coexistence of two different $R A S$ mutations in $28 \%$ of cases. We identified the coexistence of different KRAS mutations as well as KRAS and NRAS mutations. Coexistence of different $K R A S^{12-13}$ mutations has been reported by others on small series [24-26]. To our knowledge, the present study is one of the first reports concerning the coexistence of mutations in codons 12-13, 61 and 146 of KRAS (17\% of cases). In addition, we demonstrated the coexistence of mutations in KRAS and NRAS, which has been reported only once in Vagaja, N.'s article [21].

Concordance of KRAS and BRAF status between primary and metastatic lesions in mCRC has been considered to be over 95\% [17-19]. To our knowledge, few studies have evaluated inter-tumoral heterogeneity of $R A S$ mutations between primary tumors and lymph nodes or distant metastatic lesions. We observed $36 \%$ of inter-tumoral heterogeneity, with RAS mutated primary tumors being WT in lymph nodes and/or distant metastatic lesions. In addition, metastatic lymph nodes may have different $R A S$ mutations as compared to primary tumors. Concerning metastatic lymph nodes, only limited data concerning KRAS mutations are available. In one study, heterogeneity in KRAS mutations between primary tumors and lymph node metastases was found in approximately $30 \%$ of cases [27]. To summarize, in some tumors, KRAS or NRAS mutations were "universal" as they were present in all macrodissected areas in primary lesions and in metastases. In other cases, we observed multiple 
subclones with mutations present only in one area or present in all areas in primary lesions but not in metastases, and they could be classified as "primary-private" and as "primary-clonal", respectively, as described by others [26] (Figure 2).

In daily practice, $R A S$ mutation testing is usually carried out on a single transparietal section of a given location with a high fraction of neoplastic cells. However, our results have shown that not all RAS mutations present in the tumor can be detected by the current sampling method. One alternative suggestion might be the preparation of a DNA mix obtained after macrodissection of multiple histologically relevant areas, as previously proposed [28]. Moreover, for patients with multiple tumor biopsies and/or surgeries, it is important to perform RAS analyses on each sample. In addition, all KRAS and NRAS mutations must be sought simultaneously in first-line testing as they are not mutually exclusive.
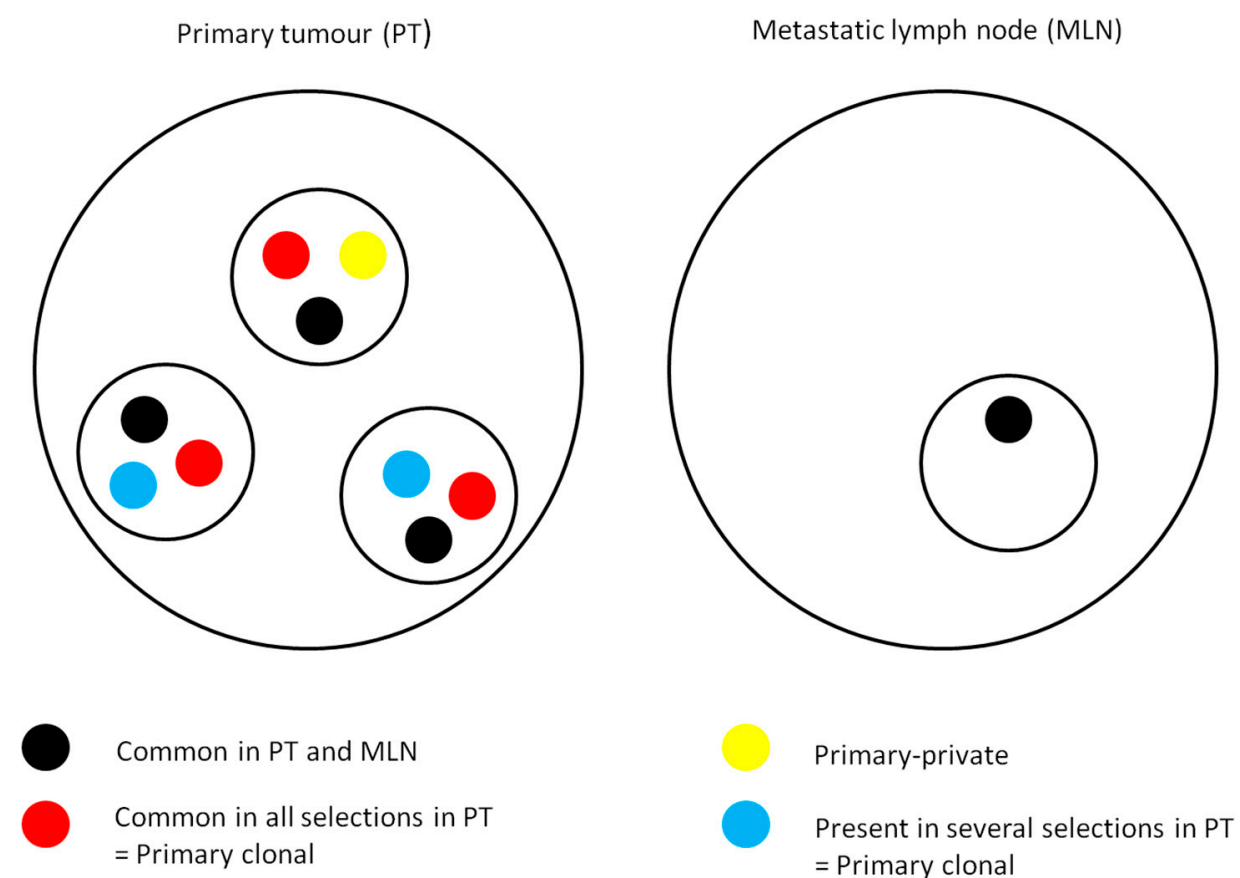

Figure 2. Subclone distribution in primary tumor and metastatic lymph node. In some cases, KRAS or NRAS mutations were universal/common as they were present in all macrodissected regions in primary tumors (PTs) and in metastatic lymph nodes (MLNs). In other cases, we observed multiple subclones with mutations present only in several regions but not all, or present in all regions in PT but not in MLN, and they could be classified as primary-clonal. In other cases, mutation was present only in one area of the tumor and could be classified as primary-private.

All of these results concerning intra- and inter-tumoral heterogeneity are dependent on the sensitivity of the technique used. The more sensitive the sequencing technique, the higher the probability of detecting minority subclones when assessing intra- and inter-tumoral heterogeneity $[8,10,29]$. However, the clinical relevance of these minority subclones called "potentially low-level mutants" is unknown (i.e., impact on anti-EGFR mAbs resistance). In a previous work, we demonstrated that low-frequency KRAS mutations (2.3\% to $10 \%)$ are associated with resistance to anti-EGFR mAbs [8]. More recently, Laurent-Puig et al., using highly sensitive picodroplet digital PCR (detection of one mutant KRAS allele in 200,000 WT KRAS alleles), suggested that patients with mCRC showing KRAS-mutated subclones lower or equal to $1 \%$ were benefiting from anti-EGFR therapies, while others were not (i.e., $>1 \%$ ) [10]. Due to the limited number of patients treated with anti-EGFR $\mathrm{mAbs}$ in our series, we cannot reliably assess the impact of intra- and inter-tumoral heterogeneity on anti-EGFR mAbs efficacy. Nevertheless, all patients with a mCRC harboring RAS PLLM and/or 
$R A S$ mutations in a limited part of the tumor (i.e., intra- or inter-tumoral heterogeneity), and treated with anti-EGFR mAbs showed disease progression $(n=5)$. To conclude, $R A S$ mutated subclones $(>1 \%)$ partially explain primary anti-EGFR $m A$ bs resistance. In contrast, secondary resistances to anti-EGFR mAbs are partially due to RAS and EGFR mutation [30-32].

One limitation of our study is the limited number of patients included, even though 78 samples from 18 tumors were analyzed. Moreover, in some cases, PPLM was found only in ITS but not in selected macrodissected areas in primary tumors. Interestingly, these results involved high tumoral cellularity cases, thereby confirming that the mutation is present but remains undetectable in the selected macrodissected areas. The major strengths of this study are: (1) analysis of extended RAS status; (2) multiple testing in primary tumors, metastatic lymph nodes and metastasis; and (3) use of a highly sensitive method.

With regard to tumor infiltration, the mutations were more frequently found in subserosa selections (pT3) and heterogeneity score increased with wall invasion; thus, pT3 selection seemed to be the best sampling zone for molecular analysis. In addition, we observed that HS was very high in several areas with a low fraction of neoplastic cells, thereby suggesting possible bias due to low tumoral cellularity or genomic amplification of the mutant allele or loss of the wild-type allele as well as mutation in both alleles [33].

\section{Materials and Methods}

\subsection{Tumor Samples}

Tumoral zone selection was performed under HES (Haematoxylin-Erythrosin-Saffron) staining by two experienced pathologists (Marion Jeantet and Gaelle Fromont) according to the 2009 pTNM classification [34]. For each tumor, selections involved the submucosa (pT1), the muscular layer (pT2), the subserosa (pT3), the metastatic lymph nodes $(\mathrm{N}+)$ and/or the visceral metastases $(\mathrm{M}+)$ when available. For instance, for a pT2 tumor, we performed pT1 and pT2 selections. For a pT3 or pT4 tumor, we performed pT1, pT2 and pT3 selections (Figure 3). The corresponding area of each tumoral zone selection was macrodissected and included in a new paraffin block. Another HES staining was performed to verify correspondence with the originally selected area. The percentage of tumor cells was assessed and selections with less than $5 \%$ of tumor cells were excluded.

A total of 60 tumoral macrodissections were analyzed. Among these samples, 15 were in pT1, 18 in pT2, 16 in pT3, 10 in N+ and 1 in M+. Four to six $10 \mu \mathrm{m}$ thick sections were used for molecular analysis.

\subsection{KRAS, NRAS and BRAF Mutation Testing}

Genomic DNA from macrodissected sections was extracted with the QIAamp DNA FFPE Tissue kit (Qiagen, Hilden, Germany). The 18 initial samples and the 60 newly macrodissected samples were tested for KRAS and NRAS mutations in codons 12-13 (KRAS ${ }^{12-13}$ and NRAS $\left.{ }^{12-13}\right)$, codon 59 (KRAS ${ }^{59}$ and $\left.N R A S^{59}\right)$, codon $61\left(K R A S^{61}\right.$ and $\left.N R A S^{61}\right)$, codon $117\left(K R A S^{117}\right.$ and NRAS $\left.{ }^{117}\right)$ and codon 146 $\left(K R A S^{146}\right.$ and $\left.N R A S^{146}\right)$. BRAF was tested for mutation in codon $600\left(B R A F^{V 600 E}\right)$. Mutations were detected by pyrosequencing using TheraScreen KRAS PyroKit CE-IVD kit (Qiagen) for KRAS ${ }^{12-13}$ and $K R A S^{61}$ or the PyroMark PCR kit (Qiagen) with homemade primers using PyroMark Assay Design 2.0 software (Qiagen, Hilden, Germany) for other RAS [35] and BRAF mutations (Table S2). PCR was carried out using $50 \mathrm{ng}$ of DNA in a total volume of $25 \mu \mathrm{L}$ according to the Qiagen supplier's instructions. Each series included a known-mutated and a known wild-type sample, as positive and negative controls. Pyrosequencing was performed using a Pyromark Q24 MDx according to the manufacturer's instructions (Qiagen). 

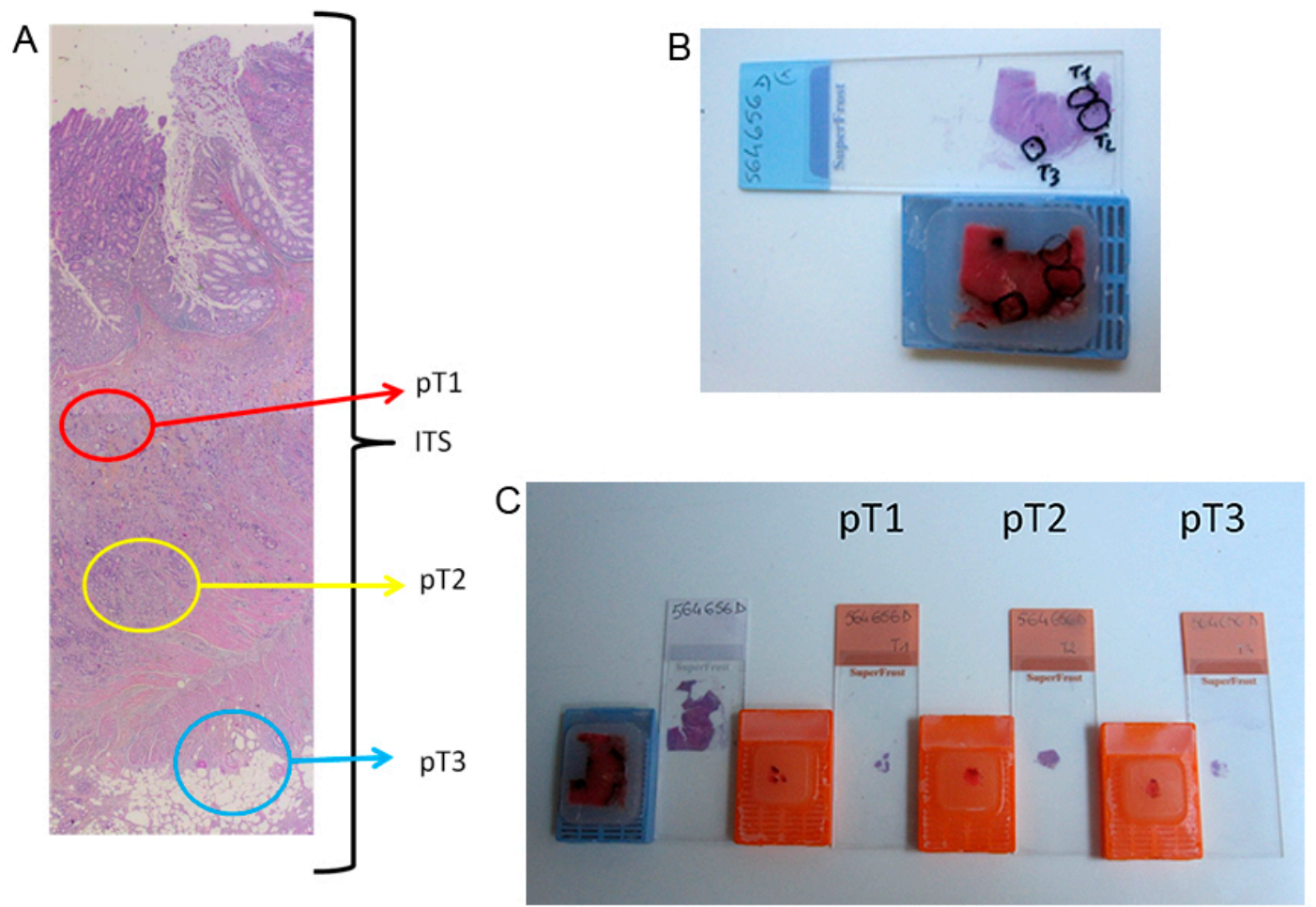

Figure 3. Tumoral selection and macrodissection. (A) Tumoral selections on the HES slide: tumoral selections on the HES slide were performed according to pathological tumor node metastasis staging (pTNM) 2009 in submucosa (pT1), in the muscular layer (pT2) for pT2 tumors, selection in submucosa, in the muscular layer and in subserosa (pT3) for pT3 and pT4 tumors; (B) tumoral selections on the HES slide and paraffin block: tumoral zones selected on the HES slide were transposed on the corresponding paraffin block; and (C) paraffin blocks and HES slides of macrodissected tumoral selections: the corresponding area of each tumoral selection was macrodissected and included in a new paraffin block. An additional HES staining was performed to ensure correspondence with the originally selected area. ITS: initial transparietal section; HES: haematoxylin-erythrosin-saffron.

\subsection{Determination of Mutant Allele Burden and Tumoral Heterogeneity}

Mutant allele frequency was determined using PyroMark Q24 2.0.6 software (Qiagen). Limit of blank (LOB) and limit of detection (LOD) were considered during biological interpretation and all samples were analyzed with regard to the LOD, which was specific to each nucleotide position [35]. Interpretation was carried out as follows: when the mutant allele frequency was $<\mathrm{LOD}$, the sample was considered as WT; when the mutant allele frequency was $\geq$ LOD and $\leq$ LOD $+3 \%$, it was considered as PLLM and when the mutant allele frequency was $>$ LOD $+3 \%$, it was considered as MUT. All mutations (PLLM and MUT) were confirmed at least twice, in two independent experiments.

Mutant allele frequency in neoplastic cells (MAFnc) and heterogeneity score (HS) were calculated as described in Normanno et al. [20]. MAFnc is the mutant allele frequency normalized for the tumor cell content. Assuming that somatic mutations are usually heterozygous events, the HS was calculated by multiplying the frequency of mutant alleles in tumor cells by two. Therefore, HS virtually corresponds to the fraction of tumor cells carrying a specific mutation. HS > 100 indicates a copy number variation, either a gain of mutant allele or a loss of WT allele.

\subsection{Statistical Analyses}

Differences in HS according to clinicopathological parameters were analyzed using the Mann-Whitney $U$ test (comparison of two groups) or Kruskal-Wallis test (comparison of more than two groups). For dichotomous variables, Fisher's exact test was used. Statistical analyses were carried 
out with a two-sided test with a significance value of 0.05 . All analyses were performed using Statview software (Statview for Windows, SAS Institute, version 5.0, Cary, NC, USA).

\section{Conclusions}

Concordance of KRAS and BRAF status between primary and metastatic lesions in mCRC has been considered to be over 95\% [17-19]. To our knowledge, the present study is one of the first reports concerning inter-tumoral heterogeneity of $R A S$ mutations between primary tumors and lymph nodes or distant metastatic lesions. In conclusion, we have demonstrated the tumoral heterogeneity of $R A S$ mutation and the co-existence of different $R A S$ mutations in CRC, which may have major clinical implications. Our results question our daily practice and expose the limits of single transparietal sampling to ensure optimal molecular analysis. Macrodissection of multiple histologically relevant areas can be an interesting solution as proposed by Richman [28]. The use of new sensitive new generation sequencing methods can also be an alternative option [36]. However, the best solution may arise from peripheral blood testing since circulating tumor cells and circulating tumor DNA are the reflection of the whole tumor $[37,38]$. These technologies provide another avenue to detect mutations, especially in patients with lymph nodes or distant metastasis.

Supplementary Materials: Supplementary materials can be found at www.mdpi.com/1422-0067/17/12/2015/s1.

Acknowledgments: Funding for this work was provided by the Ligue contre le Cancer of Vienne and of Deux-Sèvres, Poitou-Charentes region, Institut National du Cancer and the "Sport et Collection" and "Rotary Club de Civray" foundations. The authors wish to thank Jeffrey Arsham, an American translator, for having reviewed and revised the original English-language text. The authors thank Vanessa Le Berre, a research secretary, for her help in editing and formatting the manuscript.

Author Contributions: Lucie Karayan-Tapon, Gaelle Fromont, and David Tougeron conceived and designed the experiments; Marion Jeantet and Céline Archambaut performed the experiments; Marion Jeantet, Gaelle Tachon, and Lucie Karayan-Tapon analyzed the data; Ulrich Cortes and Gaelle Tachon contributed reagents/materials/analysis tools; Marion Jeantet, David Tougeron, Gaelle Tachon, and Lucie Karayan-Tapon wrote the paper.

Conflicts of Interest: The authors declare no conflict of interest. The founding sponsors had no role in the design of the study; in the collection, analyses, or interpretation of data; in the writing of the manuscript, and in the decision to publish the results.

\section{References}

1. Siegel, R.L.; Miller, K.D.; Jemal, A. Cancer statistics, 2016. CA Cancer J. Clin. 2016, 66, 7-30. [CrossRef] [PubMed]

2. Cunningham, D.; Humblet, Y.; Siena, S.; Khayat, D.; Bleiberg, H.; Santoro, A.; Bets, D.; Mueser, M.; Harstrick, A.; Verslype, C.; et al. Cetuximab monotherapy and cetuximab plus irinotecan in irinotecan-refractory metastatic colorectal cancer. N. Engl. J. Med. 2004, 351, 337-345. [CrossRef] [PubMed]

3. Douillard, J.Y.; Oliner, K.S.; Siena, S.; Tabernero, J.; Burkes, R.; Barugel, M.; Humblet, Y.; Bodoky, G.; Cunningham, D.; Jassem, J.; et al. Panitumumab-FOLFOX4 treatment and RAS mutations in colorectal cancer. N. Engl. J. Med. 2013, 369, 1023-1034. [CrossRef] [PubMed]

4. Van Cutsem, E.; Lenz, H.J.; Kohne, C.H.; Heinemann, V.; Tejpar, S.; Melezinek, I.; Beier, F.; Stroh, C.; Rougier, P.; van Krieken, J.H.; et al. Fluorouracil, leucovorin, and irinotecan plus cetuximab treatment and RAS mutations in colorectal cancer. J. Clin. Oncol. 2015, 33, 692-700. [CrossRef] [PubMed]

5. Lievre, A.; Bachet, J.B.; le Corre, D.; Boige, V.; Landi, B.; Emile, J.F.; Cote, J.F.; Tomasic, G.; Penna, C.; Ducreux, M.; et al. KRAS mutation status is predictive of response to cetuximab therapy in colorectal cancer. Cancer Res. 2006, 66, 3992-3995. [CrossRef] [PubMed]

6. Sorich, M.J.; Wiese, M.D.; Rowland, A.; Kichenadasse, G.; McKinnon, R.A.; Karapetis, C.S. Extended $R A S$ mutations and anti-EGFR monoclonal antibody survival benefit in metastatic colorectal cancer: A meta-analysis of randomized, controlled trials. Ann. Oncol. 2015, 26, 13-21. [CrossRef] [PubMed] 
7. Rowland, A.; Dias, M.M.; Wiese, M.D.; Kichenadasse, G.; McKinnon, R.A.; Karapetis, C.S.; Sorich, M.J. Meta-analysis of BRAF mutation as a predictive biomarker of benefit from anti-EGFR monoclonal antibody therapy for RAS wild-type metastatic colorectal cancer. Br. J. Cancer 2015, 112, 1888-1894. [CrossRef] [PubMed]

8. Tougeron, D.; Lecomte, T.; Pages, J.C.; Villalva, C.; Collin, C.; Ferru, A.; Tourani, J.M.; Silvain, C.; Levillain, P.; Karayan-Tapon, L. Effect of low-frequency KRAS mutations on the response to anti-EGFR therapy in metastatic colorectal cancer. Ann. Oncol. 2013, 24, 1267-1273. [CrossRef] [PubMed]

9. Wheeler, D.L.; Dunn, E.F.; Harari, P.M. Understanding resistance to EGFR inhibitors-impact on future treatment strategies. Nat. Rev. Clin. Oncol. 2010, 7, 493-507. [CrossRef] [PubMed]

10. Laurent-Puig, P.; Pekin, D.; Normand, C.; Kotsopoulos, S.K.; Nizard, P.; Perez-Toralla, K.; Rowell, R.; Olson, J.; Srinivasan, P.; le Corre, D.; et al. Clinical relevance of KRAS-mutated subclones detected with picodroplet digital PCR in advanced colorectal cancer treated with anti-EGFR therapy. Clin. Cancer Res. 2015, 21, 1087-1097. [CrossRef] [PubMed]

11. Greaves, M.; Maley, C.C. Clonal evolution in cancer. Nature 2012, 481, 306-313. [CrossRef] [PubMed]

12. Blanco-Calvo, M.; Concha, A.; Figueroa, A.; Garrido, F.; Valladares-Ayerbes, M. Colorectal Cancer Classification and Cell Heterogeneity: A Systems Oncology Approach. Int. J. Mol. Sci. 2015, 16, 13610-13632. [CrossRef] [PubMed]

13. Taniguchi, K.; Okami, J.; Kodama, K.; Higashiyama, M.; Kato, K. Intratumor heterogeneity of epidermal growth factor receptor mutations in lung cancer and its correlation to the response to gefitinib. Cancer Sci. 2008, 99, 929-935. [CrossRef] [PubMed]

14. Yancovitz, M.; Litterman, A.; Yoon, J.; Ng, E.; Shapiro, R.L.; Berman, R.S.; Pavlick, A.C.; Darvishian, F.; Christos, P.; Mazumdar, M.; et al. Intra- and inter-tumor heterogeneity of $B R A F^{V 600 E}$ mutations in primary and metastatic melanoma. PLoS ONE 2012, 7, e29336. [CrossRef] [PubMed]

15. Katona, T.M.; Jones, T.D.; Wang, M.; Eble, J.N.; Billings, S.D.; Cheng, L. Genetically heterogeneous and clonally unrelated metastases may arise in patients with cutaneous melanoma. Am. J. Surg. Pathol. 2007, 31, 1029-1037. [CrossRef] [PubMed]

16. Rajagopalan, H.; Bardelli, A.; Lengauer, C.; Kinzler, K.W.; Vogelstein, B.; Velculescu, V.E. Tumorigenesis: RAF/RAS oncogenes and mismatch-repair status. Nature 2002, 418, 934. [CrossRef] [PubMed]

17. Brannon, A.R.; Vakiani, E.; Sylvester, B.E.; Scott, S.N.; McDermott, G.; Shah, R.H.; Kania, K.; Viale, A.; Oschwald, D.M.; Vacic, V.; et al. Comparative sequencing analysis reveals high genomic concordance between matched primary and metastatic colorectal cancer lesions. Genome Biol. 2014, 15, 454. [CrossRef] [PubMed]

18. Santini, D.; Loupakis, F.; Vincenzi, B.; Floriani, I.; Stasi, I.; Canestrari, E.; Rulli, E.; Maltese, P.E.; Andreoni, F.; Masi, G.; et al. High concordance of KRAS status between primary colorectal tumors and related metastatic sites: Implications for clinical practice. Oncologist 2008, 13, 1270-1275. [CrossRef] [PubMed]

19. Baldus, S.E.; Schaefer, K.L.; Engers, R.; Hartleb, D.; Stoecklein, N.H.; Gabbert, H.E. Prevalence and heterogeneity of KRAS, BRAF, and PIK3CA mutations in primary colorectal adenocarcinomas and their corresponding metastases. Clin. Cancer Res. 2010, 16, 790-799. [CrossRef] [PubMed]

20. Normanno, N.; Rachiglio, A.M.; Lambiase, M.; Martinelli, E.; Fenizia, F.; Esposito, C.; Roma, C.; Troiani, T.; Rizzi, D.; Tatangelo, F.; et al. Heterogeneity of KRAS, NRAS, BRAF and PIK3CA mutations in metastatic colorectal cancer and potential effects on therapy in the CAPRI GOIM trial. Ann. Oncol. 2015, 26, 1710-1714. [CrossRef] [PubMed]

21. Vagaja, N.N.; Parry, J.; McCallum, D.; Thomas, M.A.; Bentel, J.M. Are all RAS mutations the same? Coexisting KRAS and NRAS mutations in a caecal adenocarcinoma and contiguous tubulovillous adenoma. J. Clin. Pathol. 2015, 68, 657-660. [CrossRef] [PubMed]

22. Sottoriva, A.; Kang, H.; Ma, Z.; Graham, T.A.; Salomon, M.P.; Zhao, J.; Marjoram, P.; Siegmund, K.; Press, M.F.; Shibata, D.; et al. A Big Bang model of human colorectal tumor growth. Nat. Genet. 2015, 47, $209-216$. [CrossRef] [PubMed]

23. Kosmidou, V.; Oikonomou, E.; Vlassi, M.; Avlonitis, S.; Katseli, A.; Tsipras, I.; Mourtzoukou, D.; Kontogeorgos, G.; Zografos, G.; Pintzas, A. Tumor heterogeneity revealed by KRAS, BRAF, and PIK3CA pyrosequencing: KRAS and PIK3CA intratumor mutation profile differences and their therapeutic implications. Hum. Mutat. 2014, 35, 329-340. [CrossRef] [PubMed] 
24. Improta, G.; Zupa, A.; Possidente, L.; Tartarone, A.; Pedicini, P.; Nappi, A.; Molinari, S.; Fraggetta, F.; Vita, G. Coexistence of two different mutations in codon 12 of the KRAS gene in colorectal cancer: Report of a case supporting the concept of tumoral heterogeneity. Oncol. Lett. 2013, 5, 1741-1743. [CrossRef] [PubMed]

25. Baisse, B.; Bouzourene, H.; Saraga, E.P.; Bosman, F.T.; Benhattar, J. Intratumor genetic heterogeneity in advanced human colorectal adenocarcinoma. Int. J. Cancer 2001, 93, 346-352. [CrossRef] [PubMed]

26. Kim, T.M.; Jung, S.H.; An, C.H.; Lee, S.H.; Baek, I.P.; Kim, M.S.; Park, S.W.; Rhee, J.K.; Lee, S.H.; Chung, Y.J. Subclonal Genomic Architectures of Primary and Metastatic Colorectal Cancer Based on Intratumoral Genetic Heterogeneity. Clin. Cancer Res. 2015, 21, 4461-4472. [CrossRef] [PubMed]

27. Oliveira, C.; Velho, S.; Moutinho, C.; Ferreira, A.; Preto, A.; Domingo, E.; Capelinha, A.F.; Duval, A.; Hamelin, R.; Machado, J.C.; et al. KRAS and BRAF oncogenic mutations in MSS colorectal carcinoma progression. Oncogene 2007, 26, 158-163. [CrossRef] [PubMed]

28. Richman, S.D.; Chambers, P.; Seymour, M.T.; Daly, C.; Grant, S.; Hemmings, G.; Quirke, P. Intra-tumoral heterogeneity of KRAS and BRAF mutation status in patients with advanced colorectal cancer (aCRC) and cost-effectiveness of multiple sample testing. Anal. Cell. Pathol. (Amst.) 2011, 34, 61-66. [CrossRef] [PubMed]

29. Molinari, F.; Felicioni, L.; Buscarino, M.; de Dosso, S.; Buttitta, F.; Malatesta, S.; Movilia, A.; Luoni, M.; Boldorini, R.; Alabiso, O.; et al. Increased detection sensitivity for KRAS mutations enhances the prediction of anti-EGFR monoclonal antibody resistance in metastatic colorectal cancer. Clin. Cancer Res. 2011, 17, 4901-4914. [CrossRef] [PubMed]

30. Misale, S.; Yaeger, R.; Hobor, S.; Scala, E.; Janakiraman, M.; Liska, D.; Valtorta, E.; Schiavo, R.; Buscarino, M.; Siravegna, G.; et al. Emergence of KRAS mutations and acquired resistance to anti-EGFR therapy in colorectal cancer. Nature 2012, 486, 532-536. [CrossRef] [PubMed]

31. Montagut, C.; Dalmases, A.; Bellosillo, B.; Crespo, M.; Pairet, S.; Iglesias, M.; Salido, M.; Gallen, M.; Marsters, S.; Tsai, S.P.; et al. Identification of a mutation in the extracellular domain of the Epidermal Growth Factor Receptor conferring cetuximab resistance in colorectal cancer. Nat. Med. 2012, 18, 221-223. [CrossRef] [PubMed]

32. Tougeron, D.; Cortes, U.; Ferru, A.; Villalva, C.; Silvain, C.; Tourani, J.M.; Levillain, P.; Karayan-Tapon, L. Epidermal growth factor receptor (EGFR) and KRAS mutations during chemotherapy plus anti-EGFR monoclonal antibody treatment in metastatic colorectal cancer. Cancer Chemother. Pharmacol. 2013, 72, 397-403. [CrossRef] [PubMed]

33. Paguirigan, A.L.; Smith, J.; Meshinchi, S.; Carroll, M.; Maley, C.; Radich, J.P. Single-cell genotyping demonstrates complex clonal diversity in acute myeloid leukemia. Sci. Transl. Med. 2015, 7, 281 re2. [CrossRef] [PubMed]

34. Gunderson, L.L.; Jessup, J.M.; Sargent, D.J.; Greene, F.L.; Stewart, A.K. Revised TN categorization for colon cancer based on national survival outcomes data. J. Clin. Oncol. 2010, 28, 264-271. [CrossRef] [PubMed]

35. Cortes, U.; Guilloteau, K.; Rouvreau, M.; Archaimbault, C.; Villalva, C.; Karayan-Tapon, L. Development of pyrosequencing methods for the rapid detection of RAS mutations in clinical samples. Exp. Mol. Pathol. 2015, 99, 207-211. [CrossRef] [PubMed]

36. Haley, L.; Tseng, L.H.; Zheng, G.; Dudley, J.; Anderson, D.A.; Azad, N.S.; Gocke, C.D.; Eshleman, J.R.; Lin, M.T. Performance characteristics of next-generation sequencing in clinical mutation detection of colorectal cancers. Mod. Pathol. 2015, 28, 1390-1399. [CrossRef] [PubMed]

37. Mohamed Suhaimi, N.-A.; Foong, Y.M.; Lee, D.Y.S.; Phyo, W.M.; Cima, I.; Lee, E.X.W.; Goh, W.L.; Lim, W.-Y.; Chia, K.S.; Kong, S.L.; et al. Non-invasive sensitive detection of KRAS and BRAF mutation in circulating tumor cells of colorectal cancer patients. Mol. Oncol. 2015, 9, 850-860. [CrossRef] [PubMed]

38. Basnet, S.; Zhang, Z.-Y.; Liao, W.-Q.; Li, S.-H.; Li, P.-S.; Ge, H.-Y. The Prognostic Value of Circulating Cell-Free DNA in Colorectal Cancer: A Meta-Analysis. J. Cancer 2016, 7, 1105-1113. [CrossRef] [PubMed]

(C) 2016 by the authors; licensee MDPI, Basel, Switzerland. This article is an open access article distributed under the terms and conditions of the Creative Commons Attribution (CC-BY) license (http://creativecommons.org/licenses/by/4.0/). 